\title{
Incomplete Caputo fractional derivative operators
}

\author{
Mehmet Ali Özarslan ${ }^{1 *}$ and Ceren Ustaoglu ${ }^{1}$
}

\section{"Correspondence:}

mehmetali.ozarslan@emu.edu.tr

'Department of Mathematics, Eastern Mediterranean University,

Gazimagusa, Turkish Republic of

Northern Cyprus

\begin{abstract}
The main aim of this paper is to give the definitions of Caputo fractional derivative operators and show their use in the special function theory. For this purpose, we introduce new types of incomplete hypergeometric functions and obtain their integral representations. Furthermore, we define incomplete Caputo fractional derivative operators and show that the images of some elementary functions under the action of incomplete Caputo fractional operators give a new type of incomplete hypergeometric functions. This definition helps us to obtain linear and bilinear generating relations for the new type incomplete Gauss hypergeometric functions.
\end{abstract}

Keywords: Incomplete Caputo fractional derivative operators; New type incomplete hypergeometric functions; Generating relations

\section{Introduction}

In recent years, some extensions of the well-known special functions have been considered by several authors (see, for example, $[4,8,9,11,15,16,18-20,31])$. The familiar incomplete gamma functions $\gamma(s, x)$ and $\Gamma(s, x)$ are defined by

$$
\gamma(s, x):=\int_{0}^{x} t^{s-1} e^{-t} d t \quad(\operatorname{Re}(s)>0 ; x \geqq 0)
$$

and

$$
\Gamma(s, x):=\int_{x}^{\infty} t^{s-1} e^{-t} d t \quad(x \geqq 0 ; \operatorname{Re}(s)>0 \text { when } x=0),
$$

respectively. They satisfy the following decomposition formula:

$$
\gamma(s, x)+\Gamma(s, x)=\Gamma(s) \quad(\operatorname{Re}(s)>0)
$$

The function $\Gamma(s)$ and its incomplete versions $\gamma(s, x)$ and $\Gamma(s, x)$ play important roles in the study of analytical solutions of a variety of problems in diverse areas of science and engineering [10].

(c) The Author(s) 2018. This article is distributed under the terms of the Creative Commons Attribution 4.0 International License (http://creativecommons.org/licenses/by/4.0/), which permits unrestricted use, distribution, and reproduction in any medium, provided you give appropriate credit to the original author(s) and the source, provide a link to the Creative Commons license, and indicate if changes were made. 
In terms of the gamma function $\Gamma(s)$, the widely used Pochhammer symbol $(\lambda)_{v}(\lambda, v \in$ C) is defined by

$$
(\lambda)_{v}:=\frac{\Gamma(\lambda+v)}{\Gamma(\lambda)}=\left\{\begin{array}{ll}
1 & (v=0 ; \lambda \in \mathbb{C} \backslash\{0\}) \\
\lambda(\lambda+1) \cdots(\lambda+v-1) & (v \in \mathbb{N} ; \lambda \in \mathbb{C})
\end{array}\right\}
$$

Recently, the incomplete Pochhammer symbols $(\lambda ; x)_{\nu}$ and $[\lambda ; x]_{\nu}(\lambda ; \nu \in \mathbb{C} ; x \geqq 0)$ were defined as follows [27]:

$$
(\lambda ; x)_{\nu}:=\frac{\gamma(\lambda+\nu, x)}{\Gamma(\lambda)} \quad(\lambda, \nu \in \mathbb{C} ; x \geqq 0)
$$

and

$$
[\lambda ; x]_{\nu}:=\frac{\Gamma(\lambda+\nu, x)}{\Gamma(\lambda)} \quad(\lambda, v \in \mathbb{C} ; x \geqq 0) .
$$

In view of (3), these incomplete Pochhammer symbols $(\lambda ; x)_{v}$ and $[\lambda ; x]_{v}$ satisfy the following decomposition relation:

$$
(\lambda ; x)_{v}+[\lambda ; x]_{v}=(\lambda)_{v} \quad(\lambda, v \in \mathbb{C} ; x \geqq 0) .
$$

The incomplete Gauss hypergeometric functions were defined by [27]

$$
2 \gamma_{1}\left[\begin{array}{lll}
(a, x) & \cdot & b ; \\
& c ;
\end{array}\right]=\sum_{n=0}^{\infty} \frac{(a ; x)_{n}(b)_{n}}{(c)_{n}} \frac{z^{n}}{n !}
$$

and

$$
{ }_{2} \Gamma_{1}\left[\begin{array}{lll}
(a, x) & \cdot & b ; \\
& c ;
\end{array}\right]=\sum_{n=0}^{\infty} \frac{[a ; x]_{n}(b)_{n}}{(c)_{n}} \frac{z^{n}}{n !} .
$$

In view of (3), these incomplete Gauss hypergeometric functions satisfy the following decomposition relation:

$$
2 \gamma_{1}\left[\begin{array}{lll}
(a, x) & b & b ; \\
& c ; & z
\end{array}\right]+{ }_{2} \Gamma_{1}\left[\begin{array}{lll}
(a, x) & b ; & b \\
& c ;
\end{array}\right]={ }_{2} F_{1}\left[\begin{array}{lll}
a & b ; & b ; \\
& c ;
\end{array}\right] .
$$

It should be also mentioned that Srivastava, Chaudhry and Agarwal [27] discussed some of their properties and some interesting applications of these families of incomplete hypergeometric functions such as integral representation, derivative formula, transformation formula, recurrence relation, and so on. Çetinkaya [7] introduced the incomplete second Appell hypergeometric functions by means of the incomplete Pochhammer symbols and obtained some integral representations and transformation formulas for these functions. After these works, incomplete hypergeometric functions have become one of the hot topics of recent years $[4,7,11,12,14,15,21,23,25,26,29-32]$.

Another approach for extending the above-mentioned hypergeometric functions was given in [17] by using the incomplete beta function defined by

$$
B_{y}(x, z):=\int_{0}^{y} t^{x-1}(1-t)^{z-1} d t, \quad \operatorname{Re}(x)>\operatorname{Re}(z)>0,0 \leq y<1 .
$$


In terms of the incomplete beta function $B_{y}(x, z)$, the incomplete Pochhammer ratios $[b, c ; y]_{n}$ and $\{b, c ; y\}_{n}$ were introduced as follows:

$$
[b, c ; y]_{n}:=\frac{B_{y}(b+n, c-b)}{B(b, c-b)}
$$

and

$$
\{b, c ; y\}_{n}:=\frac{B_{1-y}(c-b, b+n)}{B(b, c-b)},
$$

where $0 \leq y<1$.

Using the incomplete Pochhammer ratios, the authors introduced incomplete Gauss, confluent, and Appell hypergeometric functions as follows:

$$
\begin{aligned}
& { }_{2} F_{1}(a,[b, c ; y] ; x):=\sum_{n=0}^{\infty}(a)_{n}[b, c ; y]_{n} \frac{x^{n}}{n !}, \quad 0 \leq y<1, \\
& { }_{2} F_{1}(a,\{b, c ; y\} ; x):=\sum_{n=0}^{\infty}(a)_{n}\{b, c ; y\}_{n} \frac{x^{n}}{n !}, \quad 0 \leq y<1, \\
& { }_{1} F_{1}([a, b ; y] ; x):=\sum_{n=0}^{\infty}[a, b ; y]_{n} \frac{x^{n}}{n !}, \quad 0 \leq y<1, \\
& { }_{1} F_{1}(\{a, b ; y\} ; x):=\sum_{n=0}^{\infty}\{a, b ; y\}_{n} \frac{x^{n}}{n !}, \quad 0 \leq y<1, \\
& F_{1}[a, b, c ; d ; x, z ; y]:=\sum_{m, n=0}^{\infty}[a, d ; y]_{m+n}(b)_{m}(c)_{n} \frac{x^{m}}{m !} \frac{z^{n}}{n !}, \quad \max \{|x|,|z|\}<1, \\
& F_{1}\{a, b, c ; d ; x, z ; y\}:=\sum_{m, n=0}^{\infty}\{a, d ; y\}_{m+n}(b)_{m}(c)_{n} \frac{x^{m}}{m !} \frac{z^{n}}{n !}, \quad \max \{|x|,|z|\}<1, \\
& F_{2}[a, b, c ; d, e ; x, z ; y]:=\sum_{m, n=0}^{\infty}(a)_{m+n}[b, d ; y]_{m}[c, e ; y]_{n} \frac{x^{m}}{m !} \frac{z^{n}}{n !}, \quad|x|+|z|<1,
\end{aligned}
$$

and

$$
F_{2}[a, b, c ; d, e ; x, z ; y]:=\sum_{m, n=0}^{\infty}(a)_{m+n}\{b, d ; y\}_{m}\{c, e ; y\}_{n} \frac{x^{m}}{m !} \frac{z^{n}}{n !}, \quad|x|+|z|<1 .
$$

Several properties of these functions, such as integral representations, derivative formulas, transformation formulas, and recurrence relations, were obtained in [17].

Fractional calculus has gained popularity especially in the last decades. Many types of fractional derivative and integral operators and their properties have been intensive research topics of nowadays (see, for example, $[1-3,5,6,13,14,16,17,20,22,24,25,28,31$, 32]). In [17], the authors defined the following incomplete Riemann-Liouville fractional derivative operators:

$$
D_{z}^{\mu}[f(z) ; y]:=\frac{z^{-\mu}}{\Gamma(-\mu)} \int_{0}^{y} f(u z)(1-u)^{-\mu-1} d u, \quad \operatorname{Re}(\mu)<0,
$$


and

$$
D_{z}^{\mu}\{f(z) ; y\}:=\frac{z^{-\mu}}{\Gamma(-\mu)} \int_{0}^{1-y} f((1-t) z) t^{-\mu-1} d t, \quad \operatorname{Re}(\mu)<0
$$

and they gave linear and bilinear generating relations for incomplete hypergeometric functions defined in (14) and (15).

The main purpose of this paper is to give definitions of Caputo fractional derivative operators and show their use in the special function theory.

In Sect. 2, we introduce new type versions of incomplete Gauss hypergeometric functions ${ }_{2} F_{1}$, the Appell hypergeometric functions $F_{1}$ and $F_{2}$, and the Lauricella hypergeometric functions $F_{D, y}^{3}$, and we obtain their integral representations. In Sect. 3, we introduce incomplete Caputo fractional derivative operators and show that the incomplete Caputo fractional derivative operators of some elementary functions give new type incomplete hypergeometric functions defined in Sect. 2. The main results are given in Sect. 4. Finally, some graphical and tabular results are added in Sect. 5 .

\section{New type incomplete hypergeometric functions}

In this section, we introduce new types of incomplete Gauss hypergeometric functions ${ }_{2} F_{1}$, the incomplete Appell's hypergeometric functions $F_{1}$ and $F_{2}$, and the incomplete Lauricella hypergeometric functions $F_{D, y}^{3}$. Throughout this paper, we assume that $0 \leq y<1$ and $m \in \mathbb{N}$.

Definition 1 New type incomplete hypergeometric functions are defined by

$$
{ }_{2} F_{1}(a,[b, c ; y] ; x):=\sum_{n=0}^{\infty} \frac{(a)_{n}(b)_{n}}{(b-m)_{n}}[b-m, c ; y]_{n} \frac{x^{n}}{n !}
$$

and

$$
{ }_{2} F_{1}(a,\{b, c ; y\} ; x):=\sum_{n=0}^{\infty} \frac{(a)_{n}(b)_{n}}{(b-m)_{n}}\{b-m, c ; y\}_{n} \frac{x^{n}}{n !}
$$

for all $|x|<1$, where $m<\operatorname{Re}(b)<\operatorname{Re}(c)$.

Definition 2 New type incomplete Appell hypergeometric functions $F_{1}$ are defined by

$$
F_{1}[a, b, c ; d ; x, z ; y]:=\sum_{n, k=0}^{\infty} \frac{(a)_{n+k}(b)_{n}(c)_{k}}{(a-m)_{n+k}}[a-m, d ; y]_{n+k} \frac{x^{n}}{n !} \frac{z^{k}}{k !}
$$

and

$$
F_{1}\{a, b, c ; d ; x, z ; y\}:=\sum_{n, k=0}^{\infty} \frac{(a)_{n+k}(b)_{n}(c)_{k}}{(a-m)_{n+k}}\{a-m, d ; y\}_{n+k} \frac{x^{n}}{n !} \frac{z^{k}}{k !}
$$

for all $|x|<1,|z|<1$, where $m<\operatorname{Re}(a)<\operatorname{Re}(d)$. 
Definition 3 New type incomplete Appell hypergeometric functions $F_{2}$ are defined by

$$
F_{2}[a, b, c ; d, e ; x, z ; y]:=\sum_{n, k=0}^{\infty} \frac{(a)_{n+k}(b)_{n}(c)_{k}}{(b-m)_{n}(c-m)_{k}}[b-m, d ; y]_{n}[c-m, e ; y]_{k} \frac{x^{n}}{n !} \frac{z^{k}}{k !}
$$

and

$$
\begin{aligned}
& F_{2}\{a, b, c ; d, e ; x, z ; y\} \\
& \quad:=\sum_{n, k=0}^{\infty} \frac{(a)_{n+k}(b)_{n}(c)_{k}}{(b-m)_{n}(c-m)_{k}}\{b-m, d ; y\}_{n}\{c-m, e ; y\}_{k} \frac{x^{n}}{n !} \frac{z^{k}}{k !}
\end{aligned}
$$

for all $|x|+|z|<1$, where $m<\operatorname{Re}(b)<\operatorname{Re}(d)$ and $m<\operatorname{Re}(c)<\operatorname{Re}(e)$.

Definition 4 New type incomplete Lauricella hypergeometric functions $F_{D, y}^{3}$ are defined by

$$
F_{D, y}^{3}[a, b, c, d ; e ; x, w, z ; y]:=\sum_{n, k, r=0}^{\infty} \frac{(a)_{n+k+r}(b)_{n}(c)_{k}(d)_{r}}{(a-m)_{n+k+r}}[a-m, e ; y]_{n+k+r} \frac{x^{n}}{n !} \frac{w^{k}}{k !} \frac{z^{r}}{r !}
$$

and

$$
F_{D, y}^{3}\{a, b, c, d ; e ; x, w, z ; y\}:=\sum_{n, k, r=0}^{\infty} \frac{(a)_{n+k+r}(b)_{n}(c)_{k}(d)_{r}}{(a-m)_{n+k+r}}\{a-m, e ; y\}_{n+k+r} \frac{x^{n}}{n !} \frac{w^{k}}{k !} \frac{z^{r}}{r !}
$$

for all $\sqrt{x}+\sqrt{w}+\sqrt{z}<1$, where $m<\operatorname{Re}(a)<\operatorname{Re}(e)$.

Note that when $m=0$, these functions reduce to the corresponding versions given by (14), (15), (18)-(21), respectively. On the other hand, in the case $y \rightarrow 1^{-}$, the functions in (24), (26), (28), and (30) are reduced to their usual versions (similarly, as $y \rightarrow 0^{+}$, the functions in (25), (27), (29), and (31) are reduced to their usual versions).

Now we start by obtaining the integral representations of the functions given in definitions (1)-(4).

Theorem 5 We have the following integral representations:

$$
\begin{aligned}
{ }_{2} F_{1}(a,[b, c ; y] ; x)= & \frac{y^{b-m}}{B(b-m, c-b+m)} \int_{0}^{1} u^{b-m-1}(1-u y)^{c-b+m-1} \\
& \times{ }_{2} F_{1}(a, b ; b-m ; x u y) d u
\end{aligned}
$$

and

$$
\begin{aligned}
{ }_{2} F_{1}(a,\{b, c ; y\} ; x)= & \frac{(1-y)^{c-b+m}}{B(b-m, c-b+m)} \int_{0}^{1} u^{c-b+m-1}(1-u(1-y))^{b-m-1} \\
& \times{ }_{2} F_{1}(a, b ; b-m ; x(1-u(1-y))) d u
\end{aligned}
$$

for all $|x|<1$, where $m<\operatorname{Re}(b)<\operatorname{Re}(c)$. 
Proof Replacing the incomplete beta function $B_{y}(b-m+n, c-b+m)$ in definition (24) by its integral representation given by (11) and interchanging the order of summation and integration, which is permissible under the conditions given in the hypothesis, we get

$$
\begin{aligned}
{ }_{2} F_{1}(a,[b, c ; y] ; x)= & \frac{1}{B(b-m, c-b+m)} \int_{0}^{y} t^{b-m-1}(1-t)^{c-b+m-1} \\
& \times \sum_{n=0}^{\infty} \frac{(a)_{n}(b)_{n}}{(b-m)_{n}} \frac{(x t)^{n}}{n !} d t
\end{aligned}
$$

which can be written as

$$
\begin{aligned}
{ }_{2} F_{1}(a,[b, c ; y] ; x)= & \frac{y^{b-m}}{B(b-m, c-b+m)} \int_{0}^{1} u^{b-m-1}(1-u y)^{c-b+m-1} \\
& \times{ }_{2} F_{1}(a, b ; b-m ; x u y) d u .
\end{aligned}
$$

Hence the proof is completed. Formula (33) can be proved in a similar way.

Theorem 6 We have the following integral representations:

$$
\begin{aligned}
F_{1}[a, b, c ; d ; x, z ; y]= & \frac{y^{a-m}}{B(a-m, d-a+m)} \int_{0}^{1} u^{a-m-1}(1-u y)^{d-a+m-1} \\
& \times F_{1}(a ; b, c ; a-m ; x u y, z u y) d u
\end{aligned}
$$

and

$$
\begin{aligned}
F_{1}\{a, b, c ; d ; x, z ; y\}= & \frac{y^{d-a+m}}{B(a-m, d-a+m)} \int_{0}^{1} u^{d-a+m-1}(1-u(1-y))^{a-m-1} \\
& \times F_{1}(a ; b, c ; a-m ; x(1-u(1-y)), z(1-u(1-y))) d u,
\end{aligned}
$$

for all $|x|<1,|z|<1$, where $m<\operatorname{Re}(a)<\operatorname{Re}(d)$.

Proof Replacing the incomplete beta function $B_{y}(a-m+n+k, d-a+m)$ in definition (26) by its integral representation given by (11), we have that

$$
\begin{aligned}
F_{1}[a, b, c ; d ; x, z ; y]= & \frac{1}{B(a-m, d-a+m)} \int_{0}^{y} t^{a-m-1}(1-t)^{d-a+m-1} \\
& \times \sum_{n, k=0}^{\infty} \frac{(a)_{n+k}(b)_{n}(c)_{k}}{(a-m)_{n+k}} \frac{(x t)^{n}}{n !} \frac{(z t)^{k}}{k !} d t, \\
= & \frac{y^{a-m}}{B(a-m, d-a+m)} \int_{0}^{1} u^{a-m-1}(1-u y)^{d-a+m-1} \\
& \times F_{1}(a ; b, c ; a-m ; x u y, z u y) d u,
\end{aligned}
$$

whence the result. Formula (35) can be proved in a similar way. 
Theorem 7 We have the following integral representations:

$$
\begin{aligned}
F_{2}[a, b, c ; d, e ; x, z ; y]= & \frac{y^{b+c-2 m}}{B(b-m, d-b+m) B(c-m, e-c+m)} \\
& \times \int_{0}^{1} \int_{0}^{1} u^{b-m-1}(1-u y)^{d-b+m-1} v^{c-m-1}(1-v y)^{e-c+m-1} \\
& \times F_{2}(a ; b, c ; b-m, c-m ; x u y, z v y) d u d v
\end{aligned}
$$

and

$$
\begin{aligned}
F_{2}\{a, b, c ; d, e ; x, z ; y\} \\
=\frac{(1-y)^{d-b+e-c+2 m}}{B(b-m, d-b+m) B(c-m, e-c+m)} \\
\quad \times \int_{0}^{1} \int_{0}^{1} u^{d-b+m-1}(1-u(1-y))^{b-m-1} v^{e-c+m-1}(1-v(1-y))^{c-m-1} \\
\quad \times F_{2}(a ; b, c ; b-m, c-m ; x(1-u(1-y)), z(1-v(1-y))) d u d v
\end{aligned}
$$

for all $|x|+|z|<1$, where $m<\operatorname{Re}(b)<\operatorname{Re}(d)$ and $m<\operatorname{Re}(c)<\operatorname{Re}(e)$.

Proof Replacing the integral representations of $B_{y}(b-m+n, d-b+m)$ and $B_{y}(c-m+$ $k, e-c+m)$ in (28), we get

$$
\begin{aligned}
F_{2}[a, b, c ; d, e ; x, z ; y]= & \frac{1}{B(b-m, d-b+m) B(c-m, e-c+m)} \\
& \times \int_{0}^{y} \int_{0}^{y} t^{b-m-1}(1-t)^{d-b+m-1} s^{c-m-1}(1-s)^{e-c+m-1} \\
& \times \sum_{n, k=0}^{\infty} \frac{(a)_{n+k}(b)_{n}(c)_{k}}{(b-m)_{n}(c-m)_{k}} \frac{(x t)^{n}}{n !} \frac{(z s)^{k}}{k !} d t d s
\end{aligned}
$$

which is equivalent to

$$
\begin{aligned}
F_{2}[a, b, c ; d, e ; x, z ; y]= & \frac{y^{b+c-2 m}}{B(b-m, d-b+m) B(c-m, e-c+m)} \\
& \times \int_{0}^{1} \int_{0}^{1} u^{b-m-1}(1-u y)^{d-b+m-1} v^{c-m-1}(1-v y)^{e-c+m-1} \\
& \times F_{2}(a ; b, c ; b-m, c-m ; x u y, z v y) d u d v .
\end{aligned}
$$

Hence the proof is completed. Formula (37) can be proved in a similar way.

Theorem 8 We have the following integral representations:

$$
\begin{aligned}
F_{D, y}^{3}[a, b, c, d ; e ; x, w, z ; y]= & \frac{y^{a-m}}{B(a-m, e-a+m)} \int_{0}^{1} u^{a-m-1}(1-u y)^{e-a+m-1} \\
& \times F_{D}^{3}(a, b, c, d ; a-m ; x u y, w u y, z u y) d u
\end{aligned}
$$


and

$$
\begin{aligned}
& F_{D, y}^{3}\{a, b, c, d ; e ; x, w, z ; y\} \\
& =\frac{(1-y)^{e-a+m}}{B(a-m, e-a+m)} \int_{0}^{1} u^{e-a+m-1}(1-u(1-y))^{a-m-1} \\
& \quad \times F_{D}^{3}(a, b, c, d ; a-m ; x u(1-y), w u(1-y), z u(1-y)) d u,
\end{aligned}
$$

for all $\sqrt{x}+\sqrt{w}+\sqrt{z}<1$, where $m<\operatorname{Re}(a)<\operatorname{Re}(e)$.

Proof Replacing the incomplete beta function $B_{y}(a-m+n+k+r, e-a+m)$ in definition (30) by its integral representation given by (11), we find that

$$
\begin{aligned}
F_{D, y}^{3}[a, b, c, d ; e ; x, w, z ; y]= & \frac{1}{B(a-m, e-a+m)} \int_{0}^{y} t^{a-m-1}(1-t)^{e-a+m-1} \\
& \times \sum_{n, k, r=0}^{\infty} \frac{(a)_{n+k+r}(b)_{n}(c)_{k}(d)_{r}}{(a-m)_{n+k+r}} \frac{(x t)^{n}}{n !} \frac{(w t)^{k}}{k !} \frac{(z t)^{r}}{r !} d t,
\end{aligned}
$$

which can be written as

$$
\begin{aligned}
F_{D, y}^{3}[a, b, c, d ; e ; x, w, z ; y]= & \frac{y^{a-m}}{B(a-m, e-a+m)} \int_{0}^{1} u^{a-m-1}(1-u y)^{e-a+m-1} \\
& \times F_{D}^{3}(a, b, c, d ; a-m ; x u y, w u y, z u y) d u,
\end{aligned}
$$

whence the result. Formula (39) can be proved in a similar way.

\section{Incomplete Caputo fractional derivative operators}

The classical Caputo fractional derivative is defined by

$$
D^{\alpha} f(z):=\frac{1}{\Gamma(m-\alpha)} \int_{0}^{z}(z-v)^{m-\alpha-1} \frac{d^{m}}{d v^{m}} f(v) d v
$$

where $m-1<\operatorname{Re}(\alpha)<m, m \in \mathbb{N}$.

Now, we introduce the incomplete Caputo fractional derivatives as

$$
C_{z}^{\alpha}[f(z) ; y]:=\frac{1}{\Gamma(m-\alpha)} \int_{0}^{y z}(z-v)^{m-\alpha-1} \frac{d^{m}}{d v^{m}} f(v) d v
$$

and

$$
C_{z}^{\alpha}\{f(z) ; y\}:=\frac{1}{\Gamma(m-\alpha)} \int_{y z}^{z}(z-v)^{m-\alpha-1} \frac{d^{m}}{d v^{m}} f(v) d v
$$

where $0 \leq y<1$ and $m-1<\operatorname{Re}(\alpha)<m, m \in \mathbf{N}$. In the case $y \rightarrow 1^{-}$, (40) reduces to the classical Caputo fractional derivative (similarly, as $y \rightarrow 0^{+},(41)$ reduces to the classical Caputo fractional derivative). 
Now, we begin our investigation by calculating the incomplete Caputo fractional derivatives of some elementary functions.

Theorem 9 Let $m-1<\operatorname{Re}(\alpha)<m$ and $\operatorname{Re}(\alpha)<\operatorname{Re}(\lambda)$. Then

$$
C_{z}^{\alpha}\left[z^{\lambda} ; y\right]=\frac{\Gamma(\lambda+1)}{\Gamma(\lambda-\alpha+1)} \frac{B_{y}(\lambda-m+1, m-\alpha)}{B(\lambda-m+1, m-\alpha)} z^{\lambda-\alpha}
$$

and

$$
C_{z}^{\alpha}\left\{z^{\lambda} ; y\right\}=\frac{\Gamma(\lambda+1)}{\Gamma(\lambda-\alpha+1)} \frac{B_{1-y}(m-\alpha, \lambda-m+1)}{B(\lambda-m+1, m-\alpha)} z^{\lambda-\alpha} .
$$

Proof By direct calculation we get

$$
\begin{aligned}
C_{z}^{\alpha}\left[z^{\lambda} ; y\right] & =\frac{1}{\Gamma(m-\alpha)} \int_{0}^{y z}(z-v)^{m-\alpha-1} \frac{d^{m}}{d v^{m}} v^{\lambda} d v \\
& =\frac{1}{\Gamma(m-\alpha)} \frac{\Gamma(\lambda+1)}{\Gamma(\lambda-m+1)} \int_{0}^{y z}(z-v)^{m-\alpha-1} v^{\lambda-m} d v \\
& =\frac{z^{\lambda-\alpha}}{\Gamma(m-\alpha)} \frac{\Gamma(\lambda+1)}{\Gamma(\lambda-m+1)} y^{\lambda-m+1} \int_{0}^{1} u^{\lambda-m}(1-u y)^{m-\alpha-1} d u \\
& =\frac{\Gamma(\lambda+1)}{\Gamma(\lambda-\alpha+1)} \frac{B_{y}(\lambda-m+1, m-\alpha)}{B(\lambda-m+1, m-\alpha)} z^{\lambda-\alpha} .
\end{aligned}
$$

Hence the proof is completed. Formula (43) can be proved in a similar way.

The next theorem expresses the incomplete Caputo fractional derivative of an analytic function.

Theorem 10 If $f(z)$ is an analytic function on the disk $|z|<\rho$ and has a power series expansion $f(z)=\sum_{n=0}^{\infty} a_{n} z^{n}$, then

$$
C_{z}^{\alpha}\left[z^{\lambda-1} f(z) ; y\right]=\frac{\Gamma(\lambda)}{\Gamma(\lambda-\alpha)} z^{\lambda-\alpha-1} \sum_{n=0}^{\infty} a_{n} \frac{(\lambda)_{n}}{(\lambda-m)_{n}} \frac{B_{y}(\lambda-m+n, m-\alpha)}{B(\lambda-m, m-\alpha)} z^{n}
$$

and

$$
C_{z}^{\alpha}\left\{z^{\lambda-1} f(z) ; y\right\}=\frac{\Gamma(\lambda)}{\Gamma(\lambda-\alpha)} z^{\lambda-\alpha-1} \sum_{n=0}^{\infty} a_{n} \frac{(\lambda)_{n}}{(\lambda-m)_{n}} \frac{B_{1-y}(m-\alpha, \lambda-m+n)}{B(\lambda-m, m-\alpha)} z^{n}
$$

where $m-1<\operatorname{Re}(\alpha)<m$.

Proof Using Theorem 9, we get

$$
\begin{aligned}
C_{z}^{\alpha}\left[z^{\lambda-1} f(z) ; y\right] & =\sum_{n=0}^{\infty} a_{n} C_{z}^{\alpha}\left[z^{\lambda+n-1} ; y\right] \\
& =\sum_{n=0}^{\infty} a_{n}\left(\frac{1}{\Gamma(m-\alpha)} \int_{0}^{y z}(z-v)^{m-\alpha-1} \frac{d^{m}}{d v^{m}} v^{\lambda+n-1} d v\right)
\end{aligned}
$$




$$
\begin{aligned}
& =\frac{\Gamma(\lambda)}{\Gamma(\lambda-\alpha)} z^{\lambda-\alpha-1} \sum_{n=0}^{\infty} a_{n} \frac{(\lambda)_{n}}{(\lambda-\alpha)_{n}} \frac{B_{y}(\lambda-m+n, m-\alpha)}{B(\lambda-m+n, m-\alpha)} z^{n} \\
& =\frac{\Gamma(\lambda)}{\Gamma(\lambda-\alpha)} z^{\lambda-\alpha-1} \sum_{n=0}^{\infty} a_{n} \frac{(\lambda)_{n}}{(\lambda-m)_{n}} \frac{B_{y}(\lambda-m+n, m-\alpha)}{B(\lambda-m, m-\alpha)} z^{n},
\end{aligned}
$$

whence the result. Formula (45) can be proved in a similar way.

The following theorems will be useful for finding the generating function relations.

Theorem 11 Let $m-1<\operatorname{Re}(\lambda-\alpha)<m<\operatorname{Re}(\lambda)$. Then

$$
C_{z}^{\lambda-\alpha}\left[z^{\lambda-1}(1-z)^{-\mu} ; y\right]=\frac{\Gamma(\lambda)}{\Gamma(\alpha)} z^{\alpha-1}{ }_{2} F_{1}(\mu,[\lambda, \alpha ; y] ; z)
$$

and

$$
C_{z}^{\lambda-\alpha}\left\{z^{\lambda-1}(1-z)^{-\mu} ; y\right\}=\frac{\Gamma(\lambda)}{\Gamma(\alpha)} z^{\alpha-1}{ }_{2} F_{1}(\mu,\{\lambda, \alpha ; y\} ; z)
$$

for $|z|<1$.

Proof Using the power series expansion of $(1-z)^{-\mu}$, we get

$$
\begin{aligned}
C_{z}^{\lambda-\alpha}\left[z^{\lambda-1}(1-z)^{-\mu} ; y\right] & =C_{z}^{\lambda-\alpha}\left[z^{\lambda-1} \sum_{n=0}^{\infty}(\mu)_{n} \frac{z^{n}}{n !} ; y\right] \\
& =\sum_{n=0}^{\infty} \frac{(\mu)_{n}}{n !} C_{z}^{\lambda-\alpha}\left[z^{\lambda+n-1} ; y\right] \\
& =\sum_{n=0}^{\infty} \frac{(\mu)_{n}}{n !} \frac{\Gamma(\lambda+n)}{\Gamma(\alpha+n)} \frac{B_{y}(\lambda-m+n, m-\lambda+\mu)}{B(\lambda-m+n, m-\lambda+\mu)} z^{\alpha+n-1} \\
& =\frac{\Gamma(\lambda)}{\Gamma(\alpha)} z^{\alpha-1} \sum_{n=0}^{\infty} \frac{(\mu)_{n}(\lambda)_{n}}{(\alpha)_{n}} \frac{B_{y}(\lambda-m+n, m-\lambda+\mu)}{B(\lambda-m+n, m-\lambda+\mu)} \frac{z^{n}}{n !} \\
& =\frac{\Gamma(\lambda)}{\Gamma(\alpha)} z^{\alpha-1} \sum_{n=0}^{\infty} \frac{(\mu)_{n}(\lambda)_{n}}{(\lambda-m)_{n}} \frac{B_{y}(\lambda-m+n, m-\lambda+\mu)}{B(\lambda-m, \alpha-\lambda+m)} \frac{z^{n}}{n !} .
\end{aligned}
$$

Using (24), we get the result. Formula (47) can be proved in a similar way.

Theorem 12 Let $m-1<\operatorname{Re}(\lambda-\alpha)<m<\operatorname{Re}(\lambda)$. Then

$$
C_{z}^{\lambda-\alpha}\left[z^{\lambda-1}(1-a z)^{-\gamma}(1-b z)^{-\beta} ; y\right]=\frac{\Gamma(\lambda)}{\Gamma(\alpha)} z^{\alpha-1} F_{1}[\lambda, \gamma, \beta ; \alpha ; a z ; b z ; y]
$$

and

$$
C_{z}^{\lambda-\alpha}\left\{z^{\lambda-1}(1-a z)^{-\gamma}(1-b z)^{-\beta} ; y\right\}=\frac{\Gamma(\lambda)}{\Gamma(\alpha)} z^{\alpha-1} F_{1}\{\lambda, \gamma, \beta ; \alpha ; a z ; b z ; y\}
$$

for $|a z|<1$ and $|b z|<1$. 
Proof Using the power series expansions of $(1-a z)^{-\gamma}$ and $(1-b z)^{-\beta}$, we get

$$
\begin{aligned}
C_{z}^{\lambda-\alpha} & {\left[z^{\lambda-1}(1-a z)^{-\gamma}(1-b z)^{-\beta} ; y\right] } \\
& =C_{z}^{\lambda-\alpha}\left[\sum_{n, k=0}^{\infty} \frac{(\gamma)_{n}}{n !} \frac{(\beta)_{k}}{k !} a^{n} b^{k} z^{\lambda+n+k-1} ; y\right] \\
& =\sum_{n, k=0}^{\infty} \frac{(\gamma)_{n}}{n !} \frac{(\beta)_{k}}{k !} a^{n} b^{k} C_{z}^{\lambda-\alpha}\left[z^{\lambda+n+k-1} ; y\right] \\
& =\sum_{n, k=0}^{\infty} \frac{(\gamma)_{n}}{n !} \frac{(\beta)_{k}}{k !} a^{n} b^{k} \frac{\Gamma(\lambda+n+k) B_{y}(\lambda-m+n+k, m-\lambda+\alpha)}{\Gamma(\lambda-m+n+k) \Gamma(m-\lambda+\alpha)} z^{\alpha+n+k-1} \\
& =\frac{\Gamma(\lambda)}{\Gamma(\alpha)} z^{\alpha-1} \sum_{n, k=0}^{\infty} \frac{(\lambda)_{n+k}(\gamma)_{n}(\beta)_{k}}{(\lambda-m)_{n+k}} \frac{B_{y}(\lambda-m+n+k, m-\lambda+\alpha)}{B(\lambda-m, m-\lambda+\alpha)} \frac{(a z)^{n}}{n !} \frac{(b z)^{k}}{k !} .
\end{aligned}
$$

Using (26), we get the result. Formula (49) can be proved in a similar way.

Theorem 13 Let $m-1<\operatorname{Re}(\lambda-\alpha)<m<\operatorname{Re}(\lambda)$. Then

$$
\begin{array}{r}
C_{z}^{\lambda-\alpha}\left[z^{\lambda-1}(1-a z)^{-\gamma}(1-b z)^{-\beta}(1-c z)^{-\mu} ; y\right] \\
=\frac{\Gamma(\lambda)}{\Gamma(\alpha)} z^{\alpha-1} F_{D, y}^{3}[\lambda, \gamma, \beta, \mu ; \alpha ; a z ; b z ; c z ; y]
\end{array}
$$

and

$$
\begin{array}{r}
C_{z}^{\lambda-\alpha}\left\{z^{\lambda-1}(1-a z)^{-\gamma}(1-b z)^{-\beta}(1-c z)^{-\mu} ; y\right\} \\
\quad=\frac{\Gamma(\lambda)}{\Gamma(\alpha)} z^{\alpha-1} F_{D, y}^{3}\{\lambda, \gamma, \beta, \mu ; \alpha ; a z ; b z ; c z ; y\}
\end{array}
$$

for $|a z|<1,|b z|<1$, and $|c z|<1$.

Proof Using the power series expansions of $(1-a z)^{-\gamma},(1-b z)^{-\beta}$, and $(1-c z)^{-\mu}$, we get

$$
\begin{aligned}
C_{z}^{\lambda-\alpha}\left[z^{\lambda-1}(1-a z)^{-\gamma}(1-b z)^{-\beta}(1-c z)^{-\mu} ; y\right] \\
=C_{z}^{\lambda-\alpha}\left[\sum_{n, k, r=0}^{\infty} \frac{(\gamma)_{n}}{n !} \frac{(\beta)_{k}}{k !} \frac{(\mu)_{r}}{r !} a^{n} b^{k} c^{r} z^{\lambda+n+k+r-1} ; y\right] \\
=\sum_{n, k, r=0}^{\infty} \frac{(\gamma)_{n}}{n !} \frac{(\beta)_{k}}{k !} \frac{(\mu)_{r}}{r !} a^{n} b^{k} c^{r} C_{z}^{\lambda-\alpha}\left[z^{\lambda+n+k+r-1} ; y\right] \\
=\sum_{n, k, r=0}^{\infty} \frac{(\gamma)_{n}}{n !} \frac{(\beta)_{k}}{k !} \frac{(\mu)_{r}}{r !} a^{n} b^{k} c^{r} \\
\quad \times \frac{\Gamma(\lambda+n+k+r) B_{y}(\lambda-m+n+k+r, m-\lambda+\alpha)}{\Gamma(\lambda-m+n+k+r) \Gamma(m-\lambda+\alpha)} z^{\alpha+n+k+r-1}
\end{aligned}
$$




$$
\begin{aligned}
= & \frac{\Gamma(\lambda)}{\Gamma(\alpha)} z^{\alpha-1} \sum_{n, k, r=0}^{\infty} \frac{(\lambda)_{n+k+r}(\gamma)_{n}(\beta)_{k}(\mu)_{r}}{(\lambda-m)_{n+k+r}} \\
& \times \frac{B_{y}(\lambda-m+n+k+r, m-\lambda+\alpha)}{B(\lambda-m, m-\lambda+\alpha)} \frac{(a z)^{n}}{n !} \frac{(b z)^{k}}{k !} \frac{(c z)^{r}}{r !} .
\end{aligned}
$$

Using (30), we get the result. Formula (51) can be proved in a similar way.

Theorem 14 Let $m-1<\operatorname{Re}(\lambda-\alpha)<m<\operatorname{Re}(\lambda)$ and $m<\operatorname{Re}(\beta)<\operatorname{Re}(\gamma)$. Then

$$
\begin{aligned}
& C_{z}^{\lambda-\alpha}\left[z^{\lambda-1}(1-z)^{-\mu}{ }_{2} F_{1}\left(\mu,[\beta, \gamma ; y] ; \frac{x}{1-z}\right) ; y\right] \\
& \quad=\frac{\Gamma(\lambda)}{\Gamma(\alpha)} z^{\alpha-1} F_{2}[\mu, \beta, \lambda ; \gamma, \alpha ; x, z ; y]
\end{aligned}
$$

and

$$
\begin{aligned}
& C_{z}^{\lambda-\alpha}\left\{z^{\lambda-1}(1-z)^{-\mu} F_{1}\left(\mu,\{\beta, \gamma ; y\} ; \frac{x}{1-z}\right) ; y\right\} \\
& =\frac{\Gamma(\lambda)}{\Gamma(\alpha)} z^{\alpha-1} F_{2}\{\mu, \beta, \lambda ; \gamma, \alpha ; x, z ; y\}
\end{aligned}
$$

for $|x|+|z|<1$.

Proof Using the power series expansion of $(1-z)^{-\mu}$, we get

$$
\begin{aligned}
C_{z}^{\lambda-\alpha} & {\left[z^{\lambda-1}(1-z)^{-\mu}{ }_{2} F_{1}\left(\mu,[\beta, \gamma ; y] ; \frac{x}{1-z}\right) ; y\right] } \\
= & C_{z}^{\lambda-\alpha}\left[z^{\lambda-1}(1-z)^{-\mu} \sum_{n=0}^{\infty} \frac{(\mu)_{n}(\beta)_{n}}{(\beta-m)_{n} n !} \frac{B_{y}(\beta-m+n, \gamma-\beta+m)}{B(\beta-m, \gamma-\beta+m)}\left(\frac{x}{1-z}\right)^{n} ; y\right] \\
= & C_{z}^{\lambda-\alpha}\left[z^{\lambda-1} \sum_{n=0}^{\infty} \frac{(\mu)_{n}(\beta)_{n}}{(\beta-m)_{n}} \frac{B_{y}(\beta-m+n, \gamma-\beta+m)}{B(\beta-m, \gamma-\beta+m)} \frac{x^{n}}{n !}(1-z)^{-\mu-n} ; y\right] \\
= & \sum_{n=0}^{\infty} \frac{(\mu)_{n}(\beta)_{n}}{(\beta-m)_{n}} \frac{B_{y}(\beta-m+n, \gamma-\beta+m)}{B(\beta-m, \gamma-\beta+m)} \frac{x^{n}}{n !} C_{z}^{\lambda-\alpha}\left[z^{\lambda-1}(1-z)^{-\mu-n} ; y\right] \\
= & \frac{\Gamma(\lambda)}{\Gamma(\alpha)} z^{\alpha-1} \sum_{n, k=0}^{\infty}\left[\frac{(\mu)_{n+k}(\beta)_{n}(\lambda)_{k}}{(\beta-m)_{n}(\lambda-m)_{k}} \frac{B_{y}(\beta-m+n, \gamma-\beta+m)}{B(\beta-m, \gamma-\beta+m)}\right. \\
& \left.\times \frac{B_{y}(\lambda-m+k, \mu-\lambda+m)}{B(\lambda-m, \mu-\lambda+m)} \frac{x^{n} n}{n !} \frac{z^{k}}{k !}\right] .
\end{aligned}
$$

Using (28), we get the result. Formula (53) can be proved in a similar way.

\section{Main results}

In this section, we give linear and bilinear generating relations for the new type incomplete Gauss hypergeometric functions by using the relations obtained in (46), (47), (48), (49), (52), and (53). 
Theorem 15 Let $m-1<\operatorname{Re}(\lambda-\alpha)<m<\operatorname{Re}(\lambda)$. Then

$$
\sum_{n=0}^{\infty} \frac{(\mu)_{n}}{n !}{ }_{2} F_{1}(\mu+n,[\lambda, \alpha ; y] ; z) t^{n}=(1-t)^{-\mu}{ }_{2} F_{1}\left(\mu,[\lambda, \alpha ; y] ; \frac{z}{1-t}\right)
$$

and

$$
\sum_{n=0}^{\infty} \frac{(\mu)_{n}}{n !}{ }_{2} F_{1}(\mu+n,\{\lambda, \alpha ; y\} ; z) t^{n}=(1-t)^{-\mu}{ }_{2} F_{1}\left(\mu,\{\lambda, \alpha ; y\} ; \frac{z}{1-t}\right)
$$

where $|z|<\min \{1,|1-t|\}$.

Proof Taking into account the identity

$$
[(1-z)-t]^{-\mu}=(1-t)^{-\mu}\left(1-\frac{z}{1-t}\right)^{-\mu}
$$

and expanding the left-hand side, we get for $|t|<|1-z|$ that

$$
\sum_{n=0}^{\infty} \frac{(\mu)_{n}}{n !}(1-z)^{-\mu}\left(\frac{t}{1-z}\right)^{n}=(1-t)^{-\mu}\left(1-\frac{z}{1-t}\right)^{-\mu}
$$

If we multiply both sides with $z^{\lambda-1}$ and apply the incomplete Caputo fractional derivative operator $C_{z}^{\lambda-\alpha}$, we get

$$
C_{z}^{\lambda-\alpha}\left[\sum_{n=0}^{\infty} \frac{(\mu)_{n} t^{n}}{n !} z^{\lambda-1}(1-z)^{-\mu-n} ; y\right]=C_{z}^{\lambda-\alpha}\left[(1-t)^{-\mu} z^{\lambda-1}\left(1-\frac{z}{1-t}\right)^{-\mu} ; y\right] .
$$

Since $|t|<|1-z|$ and $\operatorname{Re}(\lambda)>\operatorname{Re}(\mu)>0$, it is possible to change the order of the summation and differentiation, so that

$$
\sum_{n=0}^{\infty} \frac{(\mu)_{n}}{n !} C_{z}^{\lambda-\alpha}\left[z^{\lambda-1}(1-z)^{-\mu-n} ; y\right] t^{n}=(1-t)^{-\mu} C_{z}^{\lambda-\alpha}\left[z^{\lambda-1}\left(1-\frac{z}{1-t}\right)^{-\mu} ; y\right] .
$$

So we get the result after using Theorem 11 on both sides. Formula (55) can be proved in a similar way.

Theorem 16 Let $m-1<\operatorname{Re}(\lambda-\alpha)<m<\operatorname{Re}(\lambda)$. Then

$$
\sum_{n=0}^{\infty} \frac{(\mu)_{n}}{n !}{ }_{2} F_{1}(\rho-n,[\lambda, \alpha ; y] ; z) t^{n}=(1-t)^{-\mu} F_{1}\left[\rho, \mu, \lambda ; \alpha ; z ; \frac{-z t}{1-t} ; y\right]
$$

and

$$
\sum_{n=0}^{\infty} \frac{(\mu)_{n}}{n !}{ }_{2} F_{1}(\rho-n,\{\lambda, \alpha ; y\} ; z) t^{n}=(1-t)_{1}^{-\mu}\left\{\rho, \mu, \lambda ; \alpha ; z ; \frac{-z t}{1-t} ; y\right\},
$$

where $|t|<\frac{1}{1+|z|}$. 


\section{Proof Considering}

$$
[1-(1-z) t]^{-\mu}=(1-t)^{-\mu}\left(1+\frac{z t}{1-t}\right)^{-\mu}
$$

and expanding the left-hand side, we get

$$
\sum_{n=0}^{\infty} \frac{(\mu)_{n}}{n !}(1-z)^{n} t^{n}=(1-t)^{-\mu}\left(1-\frac{-z t}{1-t}\right)^{-\mu}
$$

for $|t|<|1-z|$. Multiplying both sides with $z^{\lambda-1}(1-z)^{-\rho}$ and applying the incomplete Caputo fractional derivative operator $C_{z}^{\lambda-\alpha}$, we get

$$
\begin{aligned}
& C_{z}^{\lambda-\alpha}\left[\sum_{n=0}^{\infty} \frac{(\mu)_{n}}{n !} z^{\lambda-1}(1-z)^{-\rho+n} t^{n} ; y\right] \\
& \quad=C_{z}^{\lambda-\alpha}\left[(1-t)^{-\mu} z^{\lambda-1}(1-z)^{-\rho}\left(1-\frac{-z t}{1-t}\right)^{-\mu} ; y\right] .
\end{aligned}
$$

Since $|z t|<|1-t|$ and $\operatorname{Re}(\lambda)>\operatorname{Re}(\alpha)>0$, it is possible to change the order of summation and differentiation:

$$
\begin{aligned}
& \sum_{n=0}^{\infty} \frac{(\mu)_{n}}{n !} C_{z}^{\lambda-\alpha}\left[z^{\lambda-1}(1-z)^{-\rho+n} ; y\right] t^{n} \\
& \quad=(1-t)^{-\mu} C_{z}^{\lambda-\alpha}\left[z^{\lambda-1}(1-z)^{-\rho}\left(1-\frac{-z t}{1-t}\right)^{-\mu} ; y\right] .
\end{aligned}
$$

So we get the result after using Theorems 11 and 12. Formula (57) can be proved in a similar way.

Theorem 17 Let $m-1<\operatorname{Re}(\rho-\gamma)<m<\operatorname{Re}(\rho)$ and $m<\operatorname{Re}(\lambda)<\operatorname{Re}(\alpha)$. Then

$$
\begin{aligned}
& \sum_{n=0}^{\infty} \frac{(\mu)_{n}}{n !}{ }_{2} F_{1}(\mu+n,[\lambda, \alpha ; y] ; z){ }_{2} F_{1}(-n,[\rho, \gamma ; y] ; x) t^{n} \\
& \quad=(1-t)^{-\mu} F_{2}\left[\mu, \lambda, \rho ; \alpha, \gamma ; z, \frac{-x t}{1-t} ; y\right]
\end{aligned}
$$

and

$$
\begin{aligned}
& \sum_{n=0}^{\infty} \frac{(\mu)_{n}}{n !}{ }_{2} F_{1}(\mu+n,\{\lambda, \alpha ; y\} ; z){ }_{2} F_{1}(-n,\{\rho, \gamma ; y\} ; x) t^{n} \\
& \quad=(1-t)^{-\mu} F_{2}\left\{\mu, \lambda, \rho ; \alpha, \gamma ; z, \frac{-x t}{1-t} ; y\right\} .
\end{aligned}
$$

Proof Replacing $t$ by $(1-x) t$ in (54) and then multiplying both sides with $x^{\rho-1}$, we get

$$
\begin{aligned}
& \sum_{n=0}^{\infty} \frac{(\mu)_{n}}{n !}{ }_{2} F_{1}(\mu+n,[\lambda, \alpha ; y] ; z) x^{\rho-1}(1-x)^{n} t^{n} \\
& \quad=x^{\rho-1}[1-(1-x) t]^{-\mu}{ }_{2} F_{1}\left(\mu,[\lambda, \alpha ; y] ; \frac{z}{1-(1-x) t}\right) .
\end{aligned}
$$


Applying the fractional derivative $C_{x}^{\rho-\gamma}$ to both sides and changing the order, we find

$$
\begin{aligned}
& \sum_{n=0}^{\infty} \frac{(\mu)_{n}}{n !}{ }_{2} F_{1}(\mu+n,[\lambda, \alpha ; y] ; z) C_{x}^{\rho-\gamma}\left[x^{\rho-1}(1-x)^{n} ; y\right] t^{n} \\
& \quad=C_{x}^{\rho-\gamma}\left[x^{\rho-1}[1-(1-x) t]^{-\mu}{ }_{2} F_{1}\left(\mu,[\lambda, \alpha ; y] ; \frac{z}{1-(1-x) t}\right) ; y\right]
\end{aligned}
$$

where $|z|<1,\left|\frac{1-x}{1-z} t\right|<1$, and $\left|\frac{z}{1-t}\right|+\left|\frac{x t}{1-t}\right|<1$. Writing the equality as

$$
\begin{aligned}
& \sum_{n=0}^{\infty} \frac{(\mu)_{n}}{n !}{ }_{2} F_{1}(\mu+n,[\lambda, \alpha ; y] ; z) C_{x}^{\rho-\gamma}\left[x^{\rho-1}(1-x)^{n} ; y\right] t^{n} \\
& \quad=(1-t)^{-\mu} C_{x}^{\rho-\gamma}\left[x^{\rho-1}\left[1-\frac{-x t}{1-t}\right]^{-\mu}{ }_{2} F_{1}\left(\mu,[\lambda, \alpha ; y] ; \frac{z}{1-\frac{-x t}{1-t}}\right) ; y\right]
\end{aligned}
$$

and using Theorems 11 and 14, we get the desired result. Formula (59) can be proved in a similar way.

\section{Graphical and tabular representation for ${ }_{2} F_{1}(a,[b, c ; y] ; x)$}

In this section, we take $m=2, a=0.8, b=3.3, c=3.5, x_{i+1}=0.001+i(0.05257895)$, $i=0,1, \ldots, 19$ and evaluate the values of ${ }_{2} F_{1}(a,[b, c ; y] ; x)$ by using Wolfram Mathematica (see Table 1). Figure 1 illustrates the graph of integral representation of the incomplete hypergeometric function ${ }_{2} F_{1}(a,[b, c ; y] ; x)$.

\section{Conclusion}

Recently, in [17], we defined incomplete Pochhammer ratios in terms of the incomplete beta function. Then, with the help of this incomplete Pochhammer ratios, we introduced new incomplete Gauss, confluent hypergeometric, and Appell's functions. Furthermore, incomplete Riemann-Liouville fractional derivative operators were introduced. In our

Table 1 Values for $y=0.4, y=0.6, y=0.8$, and $y=1$

\begin{tabular}{lllll}
\hline$x_{i}$ & $y=0.4$ & $y=0.6$ & $y=0.8$ & $y=1$ \\
\hline$x_{1}$ & 0.579398 & 0.816125 & 0.958368 & 1.00075 \\
$x_{2}$ & 0.602232 & 0.848289 & 0.996139 & 1.0402 \\
$x_{3}$ & 0.62723 & 0.8835 & 1.03749 & 1.08337 \\
$x_{4}$ & 0.65473 & 0.92236 & 1.08297 & 1.13087 \\
$x_{5}$ & 0.685151 & 0.965086 & 1.13329 & 1.18342 \\
$x_{6}$ & 0.71901 & 1.01278 & 1.1893 & 1.2419 \\
$x_{7}$ & 0.756961 & 1.06624 & 1.25207 & 1.30745 \\
$x_{8}$ & 0.799839 & 1.12663 & 1.323 & 1.38151 \\
$x_{9}$ & 0.848732 & 1.1955 & 1.40387 & 1.46596 \\
$x_{10}$ & 0.905085 & 1.27488 & 1.49708 & 1.56329 \\
$x_{11}$ & 0.970863 & 1.36753 & 1.60588 & 1.67691 \\
$x_{12}$ & 1.04882 & 1.47734 & 1.73483 & 1.81155 \\
$x_{13}$ & 1.14294 & 1.60991 & 1.8905 & 1.97412 \\
$x_{14}$ & 1.25923 & 1.77372 & 2.08286 & 2.17499 \\
$x_{15}$ & 1.40729 & 1.98227 & 2.32776 & 2.43071 \\
$x_{16}$ & 1.60346 & 2.25859 & 2.65224 & 2.76954 \\
$x_{17}$ & 1.87841 & 2.64588 & 3.10703 & 3.24445 \\
$x_{18}$ & 2.29816 & 3.23713 & 3.80134 & 3.96946 \\
$x_{19}$ & 3.0403 & 4.28248 & 5.02888 & 5.2513 \\
$x_{20}$ & 4.85056 & 6.83238 & 8.0232 & 8.37805 \\
\hline
\end{tabular}


Figure 1 Integral representation of the incomplete hypergeometric function ${ }_{2} F_{1}(a,[b, c ; y] ; x)$

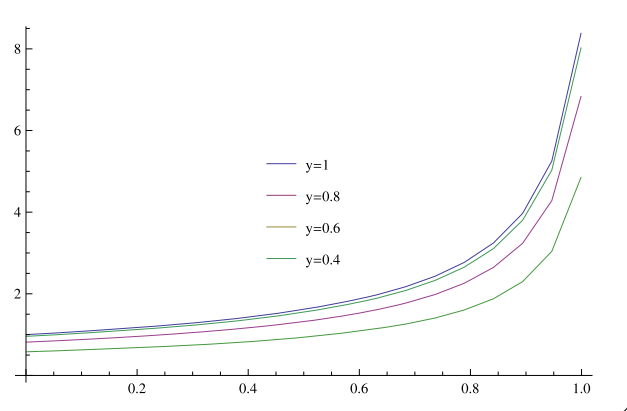

present investigation, we give definitions of an incomplete Caputo fractional derivative operator. To show the use of this incomplete Caputo fractional derivative operator, we introduce new type versions of incomplete Gauss, Appell, and Lauricella hypergeometric functions. Finally, we obtain linear and bilinear generating relations for the new type versions of incomplete hypergeometric functions.

Some advantages of the study are as follows:

1. The usual Caputo fractional derivative operator is singular, but the incomplete Caputo fractional derivative operator

$$
\begin{aligned}
& C_{z}^{\alpha}[f(z) ; y]=\frac{1}{\Gamma(\mu-\alpha)} \int_{0}^{y z}(z-v)^{m-\alpha-1} \frac{d^{m}}{d \nu^{m}} f(\nu) d \nu \\
& 0 \leq y<1, m-1<\operatorname{Re}(\alpha)<m
\end{aligned}
$$

is nonsingular.

2. The incomplete hypergeometric functions were defined in [27] by (8) and (9).

Fractional calculus properties of these functions cannot be given. Furthermore, the generating functions cannot be obtained by using the fractional calculus approach. In this paper, we solved these problems with the help of our new definitions.

We believe that the approach used here to define the incomplete hypergeometric functions will be used in the definitions of multivariable incomplete hypergeometric functions. In the study of their fractional calculus properties the new incomplete Caputo fractional derivative operators will be the main tools. Finally, we believe that these new defined hypergeometric functions and incomplete Caputo fractional derivative operators will be potentially useful in engineering and applied sciences and will help to investigate interesting phenomena arising in the real-world problems.

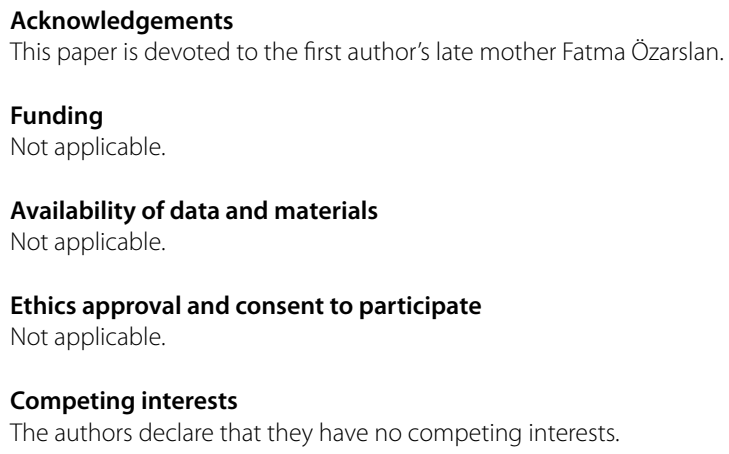




\section{Consent for publication}

Not applicable.

\section{Authors' contributions}

Both authors have read the manuscript and agreed to its content and are responsible for all aspects of the accuracy and integrity of the manuscript. The authors declare that each author equally contributed to this paper.

\section{Publisher's Note}

Springer Nature remains neutral with regard to jurisdictional claims in published maps and institutional affiliations.

\section{Received: 5 December 2017 Accepted: 24 May 2018 Published online: 15 June 2018}

\section{References}

1. Agarwal, P.: Some inequalities involving Hadamard-type k-fractional integral operators. Math. Methods Appl. Sci. 40, 3882-3891 (2017)

2. Agarwal, P., Choi, J.: Fractional calculus operators and their image formulas. J. Korean Math. Soc. 53, 1183-1210 (2016)

3. Agarwal, P., Jain, S., Mansour, T.: Further extended Caputo fractional derivative operator and its applications. Russ. J. Math. Phys. 24, 415-425 (2017)

4. Agarwal, R.P., Luo, M.J., Agarwal, P.: On the extended Appell-Lauricella hypergeometric functions and their applications. Filomat 31, 3693-3713 (2017)

5. Baleanu, D., Kumar, D., Purohit, S.D.: Generalized fractional integrals of product of two $H$-functions and a general class of polynomials. Int. J. Comput. Math. 93, 1320-1329 (2016)

6. Baleanu, D., Purohit, S.D., Prajapati, J.C.: Integral inequalities involving generalized Erdelyi-Kober fractional integral operators. Open Math. 14, 89-99 (2016)

7. Çetinkaya, A.: The incomplete second Appell hypergeometric functions. Appl. Math. Comput. 219, 8332-8337 (2013)

8. Chaudhry, M.A., Qadir, A., Rafique, M., Zubair, S.M.: Extension of Euler's beta function. J. Comput. Appl. Math. 78, 19-32 (1997)

9. Chaudhry, M.A., Qadir, A., Srivastava, H.M., Paris, R.B.: Extended hypergeometric and confluent hypergeometric functions. Appl. Math. Comput. 159, 589-602 (2004)

10. Chaudry, M.A., Zubair, S.M.: On a class of incomplete gamma functions with applications. Dhahran, Saudi Arabia (2001)

11. Cho, N.E., Srivastava, R.: Some extended Pochhammer symbols and their applications involving generalized hypergeometric polynomials. Appl. Math. Comput. 234, 277-285 (2014)

12. Choi, J., Agarwal, P.: Certain class of generating functions for the incomplete hypergeometric functions. Abstr. Appl. Anal. 2014, Article ID 714560 (2014)

13. Kıymaz, I.O., Agarwal, P., Jain, S., Çetinkaya, A.: On a new extension of Caputo fractional derivative operator. In Advances in Real and Complex Analysis with Applications, pp. 261-275 (2017). https://doi.org/10.1007/978-981-10-4337-6_11

14. Kıymaz, I.O., Çetinkaya, A., Agarwal, P.: An extension of Caputo fractional derivative operator and its applications. J. Nonlinear Sci. Appl. 9, 3611-3621 (2016)

15. Lin, S.D., Srivastava, H.M., Wong, M.M.: Some applications of Srivastava's theorem involving a certain family of generalized and extended hypergeometric polynomials. Filomat 29, 1811-1819 (2015)

16. Özarslan, M.A., Özergin, E.: Some generating relations for extended hypergeometric functions via generalized fractional derivative operator. Math. Comput. Model. 52, 1825-1833 (2010)

17. Özarslan, M.A., Ustaoglu, C.: Incomplete Riemann-Liouville fractional derivative operators and incomplete hypergeometric functions (submitted)

18. Özergin, E.: Some properties of hypergeometric functions. Dissertation, Eastern Mediterranean University (2011)

19. Özergin, E., Özarslan, M.A., Altın, A.: Extension of gamma, beta and hypergeometric functions. J. Comput. Appl. Math. 235, 4601-4610 (2011)

20. Parmar, R.K.: Some generating relations for generalized extended hypergeometric functions involving generalized fractional derivative operator. J. Concr. Appl. Math. 12, 217-228 (2014)

21. Parmar, R.K., Saxena, R.K.: The incomplete generalized tau-hypergeometric and second tau-Appell functions. J. Korean Math. Soc. 53, 363-379 (2016)

22. Rahman, G., Baleanu, D., Al-Qurashi, M., Purohit, S.D., Mubeen, S., Arshad, M.: The extended Mittag-Leffler function via fractional calculus. J. Nonlinear Sci. Appl. 10,4244-4253 (2017)

23. Sahai, V., Verma, A.: On an extension of the generalized Pochhammer symbol and its applications to hypergeometric functions. Asian-Eur. J. Math. 9, 1-11 (2016)

24. Srivastava, H.M., Agarwal, P., Jain, S.: Generating functions for the generalized Gauss hypergeometric functions. Appl. Math. Comput. 247, 348-352 (2014)

25. Srivastava, H.M., Agarwal, R., Jain, S.: Integral transform and fractional derivative formulas involving the extended generalized hypergeometric functions and probability distributions. Math. Methods Appl. Sci. 40, 255-273 (2017)

26. Srivastava, H.M., Çetinkaya, A., Kıymaz, I.O.: A certain generalized Pochhammer symbol and its applications to hypergeometric functions. Appl. Math. Comput. 226, 484-491 (2014)

27. Srivastava, H.M., Chaudhry, M.A., Agarwal, R.P.: The incomplete Pochhammer symbols and their applications to hypergeometric and related functions. Integral Transforms Spec. Funct. 23, 659-683 (2012)

28. Srivastava, H.M., Manocha, H.L.: A Treatise on Generating Functions. Halsted, New York (1984)

29. Srivastava, R.: Some properties of a family of incomplete hypergeometric functions. Russ. J. Math. Phys. 20, 121-128 (2013)

30. Srivastava, R.: Some generalizations of Pochhammer's symbol and their associated families of hypergeometric functions and hypergeometric polynomials. Appl. Math. Inf. Sci. 7, 2195-2206 (2013) 
31. Srivastava, R.: Some classes of generating functions associated with a certain family of extended and generalized hypergeometric functions. Appl. Math. Comput. 243, 132-137 (2014)

32. Srivastava, R., Cho, N.E.: Generating functions for a certain class of hypergeometric polynomials. Appl. Math. Comput. 219, 3219-3225 (2012)

Submit your manuscript to a SpringerOpen ${ }^{\circ}$ journal and benefit from:

- Convenient online submission

$\checkmark$ Rigorous peer review

Open access: articles freely available online

- High visibility within the field

- Retaining the copyright to your article

Submit your next manuscript at $\gg$ springeropen.com 